\title{
Measurement of Pancreatic Fat Fraction by CT Histogram Analysis to Predict Pancreatic Fistula after Pancreaticoduodenectomy
}

\author{
Wonju Hong, $\mathrm{MD}^{1}$, Hong Il Ha, MD, PhD ${ }^{1}$, Jung Woo Lee, $M D^{2}$, Sang Min Lee, MD, PhD', \\ Min-Jeong Kim, MD ${ }^{1}$ \\ Departments of ${ }^{1}$ Radiology and ${ }^{2}$ Surgery, Hallym University Sacred Heart Hospital, Anyang, Korea
}

Objective: To evaluate the effectiveness of computed tomography (CT) Hounsfield unit histogram analysis (HUHA) in postoperative pancreatic fistula (PF) prediction.

Materials and Methods: Fifty-four patients (33 males and 21 females; mean age, 65.6 years; age range, 37-89 years) who had undergone preoperative CT and pancreaticoduodenectomy were included in this retrospective study. Two radiologists measured mean CT Hounsfield unit (CTHU) values by drawing regions of interest (ROIs) at the level of the pancreaticojejunostomy site on preoperative pre-contrast images. The HUHA values were arbitrarily divided into three categories, comprising HUHA-A $\leq 0 \mathrm{HU}, 0 \mathrm{HU}<\mathrm{HUHA}-\mathrm{B}<30 \mathrm{HU}$, and HUHA-C $\geq 30 \mathrm{HU}$. Each HUHA value within the ROI was calculated as a percentage of the entire area using commercial 3-dimensional analysis software. Pancreas texture was evaluated as soft or hard by manual palpation.

Results: Fifteen patients $(27.8 \%)$ had clinically relevant PFs. The PF group had significantly higher HUHA-A $(p<0.01)$ and significantly lower mean CTHU $(p<0.01)$ values than those of the non-PF group. The HUHA-A value had a moderately strong correlation with PF occurrence $(r=0.60, p<0.01)$, whereas the mean CTHU had a weak negative correlation with PF occurrence $(r=-0.27, p<0.01)$. The HUHA-A and mean CTHU areas under the curve (AUCs) for predicting PF occurrence were 0.86 and 0.65 , respectively, with significant difference $(p<0.01)$. The HUHA-A and mean CTHU AUCs for predicting pancreatic softness were 0.86 and 0.64 , respectively, with significant difference $(p<0.01)$.

Conclusion: The HUHA-A values on preoperative pre-contrast CT images demonstrate a strong correlation with PF occurrence. Keywords: Pancreaticoduodenectomy; Pancreatic fistula; MDCT; Histogram analysis; Attenuation; Pancreatic softness

\section{INTRODUCTION}

Pancreaticoduodenectomy (PD) is the main treatment for periampullary and pancreatic head cancers (1-3). Postoperative mortality after PD has decreased significantly over the past decade $(4,5)$. However, the incidence of

Received August 16, 2018; accepted after revision January 11, 2019.

Corresponding author: Hong Il Ha, MD, PhD, Department of Radiology, Hallym University Sacred Heart Hospital, 22 Gwanpyeong-ro 170beon-gil, Dongan-gu, Anyang 14068, Korea.

- Tel: (8231) 380-3895 - Fax: (8231) 380-3878

-E-mail: ha.hongil@gmail.com

This is an Open Access article distributed under the terms of the Creative Commons Attribution Non-Commercial License (https://creativecommons.org/licenses/by-nc/4.0) which permits unrestricted non-commercial use, distribution, and reproduction in any medium, provided the original work is properly cited. morbidity still remains high, ranging from $16 \%$ to $41 \%$ (6). Postoperative pancreatic fistula (PF) is a major complication as well as a major factor contributing to increased morbidity, with incidences ranging from $5 \%$ to $40 \%$ (6-10). Pancreatic softness is the most frequently reported risk factor for PF. The presence of a fatty pancreas on microscopic pathological examination constitutes a risk factor for PF $(9,11-13)$. Therefore, evaluating fatty infiltration of the pancreas on preoperative imaging may be useful for predicting PF and considering alternative treatment strategies (14).

Mean computed tomography attenuation in Hounsfield unit (CTHU) on nonenhanced images can reflect pancreatic fatty changes, and a low pancreatic attenuation index is strongly associated with PF (15). However, since the mean CTHU value represents an average value of all 
tissues, including fat, water, and pancreatic parenchyma, it is limited in terms of assessing the amount of fat in the pancreatic parenchyma. Recently, commercial threedimensional (3D) imaging analysis software has made it possible to classify CTHU values in regions of interest (ROIs) within a specific range of $\mathrm{HU}$ values (16). We hypothesized that the negative CTHU fraction in the pancreatic parenchymal ROI represented the amount of fat in the pancreatic parenchyma. The purpose of this study was to evaluate the effectiveness of CTHU histogram analysis (HUHA) in predicting PF occurrence and pancreatic softness.

\section{MATERIALS AND METHODS}

\section{Patients}

This retrospective study was approved by our Institutional Review Board of Hallym University Sacred Heart Hospital, and informed consent was waived. The study included 69 patients who underwent PD between January 2015 and July 2018 and were selected using "pancreaticoduodenectomy" as the search term in the hospital's electronic medical record system. Only patients with a preoperative CT scan available for review that was taken within 30 days before surgery were included in the study. Of these patients, 15 were excluded due to a lack of available preoperative CT scans $(n=6)$ or measurable pancreatic parenchyma $(n=4)$ or having underwent total pancreatectomy $(n=3)$ or bypass gastrojejunostomy $(n=2)$ instead of PD. In total, 54 patients ( 33 males and 21 females; mean age, 65.6 years; age range, 37-89 years) were included (Fig. 1). None of the patients received preoperative chemotherapy or radiation therapy. The clinical laboratory data for each patient were

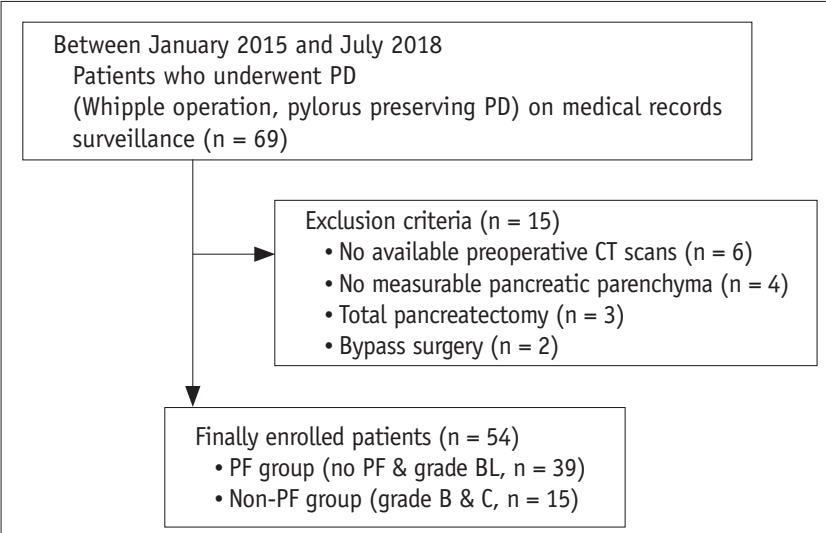

Fig. 1. Patient selection flowchart. $B L=$ biochemical fistula, $C T=$ computed tomography, $\mathrm{PD}=$ pancreaticoduodenectomy, $\mathrm{PF}=$ pancreatic fistula analyzed using previously described methods $(17,18)$.

\section{PF Definition, Surgical Procedure, and Texture Analyses}

PF was classified according to the 2016 update of the International Study Group on Pancreatic Fistula (ISGPF) as any measurable drainage output from an intraoperatively placed drain, or a postoperatively placed percutaneous drain, on or after postoperative day 3 with an amylase content greater than three times the upper limit of the normal serum amylase level ( $>300 \mathrm{U} / \mathrm{L}$ ). The severity of the fistula was graded as biochemical fistula (BL), $B$, or $C$ as follows: 1 ) grade $B L$ represents a transient, asymptomatic fistula with elevated drain amylase levels only, which does not require treatment or deviation from clinical management; 2) grade B represents a symptomatic, clinically apparent fistula requiring diagnostic elevation and therapeutic management, such as percutaneous or endoscopic drainage or an angiographic procedure for bleeding; and 3) grade $C$ represents a severe, clinically significant fistula requiring a major deviation in clinical management, such as reoperation, organ failure, or death $(8,10)$. Grades $B$ and $C$ are clinically important since they require altered postoperative management and interventions, whereas grade $\mathrm{BL}$ only requires observation $(18,19)$. Therefore, we combined grades $B$ and $C$ into the clinically significant PF group and grade BL and no PF into the non-PF group.

The texture of the pancreatic parenchyma was assessed as soft or hard by manual palpation by a single surgeon.

\section{CT Acquisition Protocol}

All CT images were obtained using one of three scanners, including a 64-slice multidetector CT (MDCT) scanner (Brilliance 64; Philips Healthcare, Cleveland, OH, USA) and two 128-slice MDCT scanners (SOMATOM Definition Edge or SOMATOM Definition Flash; Siemens Healthineers, Forchheim, Germany). Automatic tube voltage selection and current modulation were used for 128 -slice MDCT scans, whereas automatic tube current modulation was used for 64-slice MDCT scans. The general scanning parameters were as follows: detector configuration, $64 \times 0.6 \mathrm{~mm}$ for 64 -slice MDCT scans and $128 \times 0.6 \mathrm{~mm}$ for 128 -slice MDCT scans; gantry rotation time of 0.5 seconds for all scanners; tube current, 140-200 $\mathrm{mAs}$; tube voltage, $100 \mathrm{kVp}$ or $120 \mathrm{kVp}$; slice thickness, $3 \mathrm{~mm}$; reconstruction interval, $3 \mathrm{~mm}$; and filtered back projection. The HUHA and mean CTHU values were measured on pre-contrast CT images to avoid contrast agent effects. 


\section{CT Image Analyses}

We evaluated the HUHA and mean CTHU values using commercial $3 \mathrm{D}$ analysis software (Aquarius iNtuition v4.4.12; TeraRecon, Foster city, CA, USA). The HUHA of the pancreas was evaluated on preoperative pre-contrast $\mathrm{CT}$ images. First, all pre-contrast $\mathrm{CT}$ images were saved in DICOM format and imported into the commercial 3D analysis software. The imported images were reconstructed as reformatted axial, sagittal, and coronal images. Referring to postoperative CT images, an observer selected one axial image containing the pancreatic parenchyma using the superior mesenteric vein (SMV) as the reference point, which corresponded to the pancreaticojejunostomy site. Following this, a large round ROI was drawn within the pancreatic parenchyma on the selected axial image. The retroperitoneal or peripancreatic fat was carefully excluded from this ROI. Using the 3D analysis software, a corresponding sagittal image was selected, and a round ROI was similarly drawn. The HUHA and mean CTHU values were automatically and simultaneously analyzed. The HUHA value was arbitrarily divided into three categories, comprising HUHA-A $\leq 0 \mathrm{HU}, 0 \mathrm{HU}<\mathrm{HUHA}-\mathrm{B}<30 \mathrm{HU}$, and HUHA-C $\geq$ $30 \mathrm{HU}$. Categories HUHA-A, HUHA-B, and HUHA-C represent the fat, water, and pancreatic parenchyma content within the ROI, respectively. Each HUHA value was calculated as a percentage of the entire area. The axial and sagittal HUHA and mean CTHU values were expressed as average values (Fig. 2).

All data measurements were performed by two observers (i.e., observer 1 with 10 years of abdominal imaging experience and observer 2, a third-year radiology resident). Other risk factors related to PF occurrence were evaluated by one observer. The pancreatic thickness and main pancreatic duct (MPD) diameter were measured at the level of the confluence of the SMV and portal vein. The thickness of the subcutaneous fat adjacent to the umbilicus as well
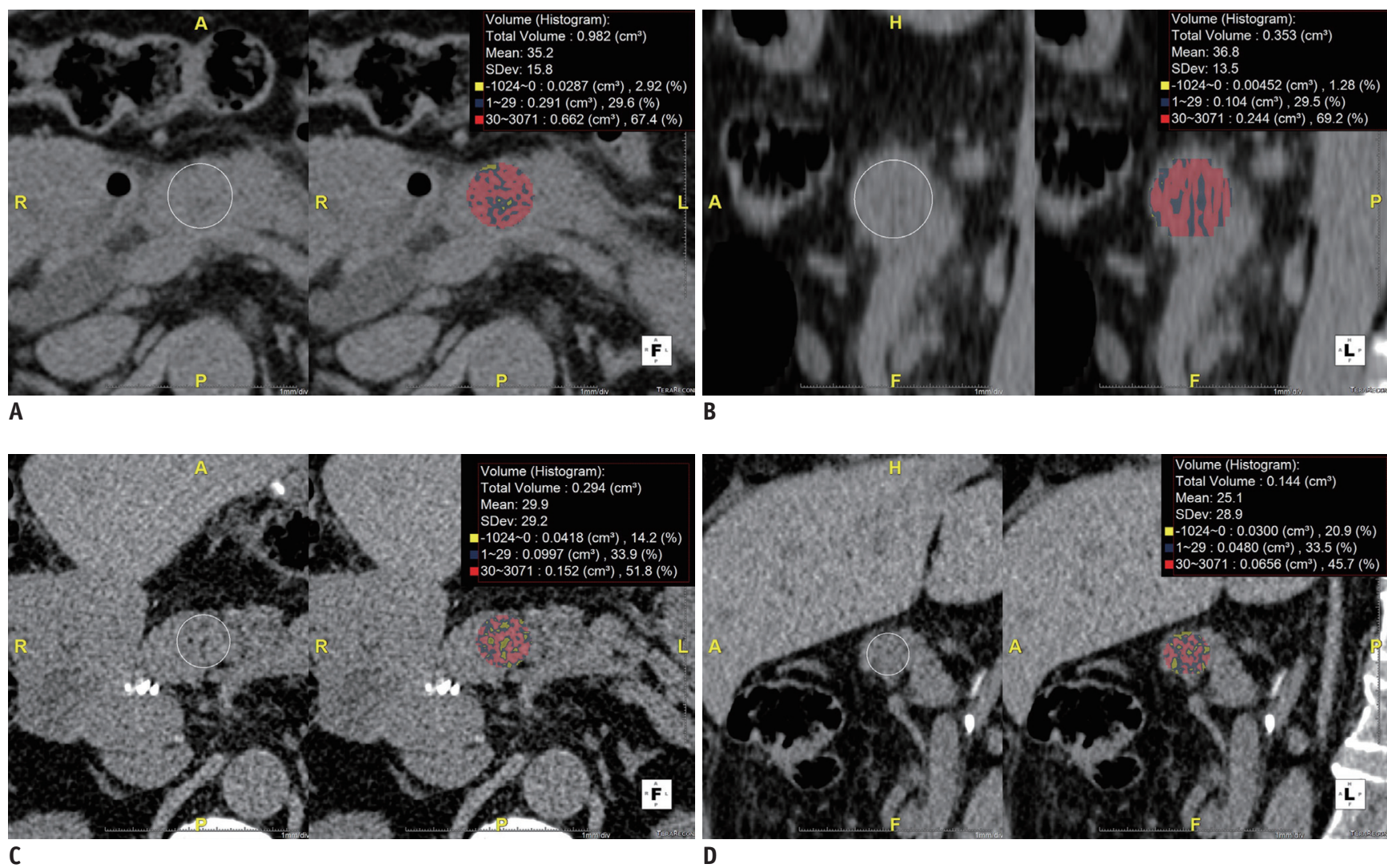

Fig. 2. Measurement of HUHA. ROIs were drawn on axial and corresponding sagittal preoperative pre-contrast CT images at level of pancreas neck near pancreaticojejunostomy site with reference to postoperative $\mathrm{CT}$ images. Three-dimensional analysis software automatically generated each HUHA-A (-1024-0 HU), HUHA-B (1-29 HU), HUHA-C (30-3071 HU), and mean CTHU value. Each HUHA value within ROI was calculated as percentage of entire area in real time.

Axial (A) and sagittal (B) images from 76-year-old male patient without PF who underwent pylorus-preserving PD for pancreatic cancer show HUHA-A of $2.1 \%$ and mean CTHU of $36 \mathrm{HU}$. Axial (C) and sagittal (D) images from 77-year-old female patient with grade B PF who underwent pylorus-preserving PD for ampulla of Vater cancer show HUHA-A of $17.6 \%$ and mean CTHU of $27.5 \mathrm{HU}$. CTHU = CT Hounsfield unit, HUHA = Hounsfield unit histogram analysis, ROI = region of interest, SD = standard deviation 
as the thickness of the perirenal fat at the level of the left renal vein were also measured on preoperative CT $(6,11$, $13,18,20)$.

\section{Statistical Analyses}

Inter-observer agreement for the HUHA and mean CTHU measurements was assessed using Bland-Altman plots, and mean differences and 95\% limits of agreement (mean difference $\pm 1.96 \times$ standard deviation) were determined. Independent-samples $t$ tests with or without Welch's test were used to compare quantitative parameters with respect to normal or unequal distributions. Chi-square tests and
McNemar's test were used to assess categorical data. A correlation coefficient was calculated to evaluate the relationship between the HUHA values and PF occurrence or pancreatic softness. Predictions of PF occurrence and pancreatic softness were analyzed with receiver operating characteristic (ROC) curves using each HUHA and mean CTHU value. ROC curves were compared using the method of Delong et al. (21), which calculated the exact binomial confidence intervals (CIs) for the areas under the curve (AUCs) (22). Statistical analyses were performed using commercial software (MedCalc Software bvba, 0stend, Belgium). $P<0.05$ indicated statistical significance.

Table 1. Demographic Findings of Study Population

\begin{tabular}{|c|c|c|c|}
\hline & PF Group $(n=15)$ & Non-PF Group $(n=39)$ & $P$ \\
\hline Age $($ mean $\pm S D, y r)$ & $67.5 \pm 11.6$ & $65.6 \pm 11.3$ & 0.71 \\
\hline Sex & & & 0.92 \\
\hline Male & 9 & 24 & \\
\hline Female & 6 & 15 & \\
\hline Diagnosis & & & 0.41 \\
\hline Pancreatic cancer & 10 & 18 & \\
\hline IPMN & 0 & 2 & \\
\hline CBD cancer & 3 & 6 & \\
\hline Duodenal cancer & 1 & 0 & \\
\hline GIST & 0 & 4 & \\
\hline Ampulla of Vater cancer & 2 & 2 & \\
\hline 0thers* & 2 & 4 & \\
\hline Body mass index $\left(\mathrm{kg} / \mathrm{m}^{2}\right)$ & $24.5 \pm 3.7$ & $22.6 \pm 2.7$ & 0.06 \\
\hline Pancreatic duct size $(\mathrm{mm})$ & $3.5 \pm 4.9$ & $3.1 \pm 2.4$ & 0.57 \\
\hline Pancreas thickness (mm) & $18.6 \pm 3.5$ & $20.9 \pm 17.9$ & 0.11 \\
\hline Left anterior pararenal fat thickness (mm) & $8.7 \pm 3.5$ & $7.8 \pm 4.1$ & 0.46 \\
\hline Subcutaneous fat thickness (mm) & $22.6 \pm 8.2$ & $20.3 \pm 7.7$ & 0.38 \\
\hline Pancreas texture classified by surgeon & & & $<0.001$ \\
\hline Soft $(n=27)$ & 12 & 15 & \\
\hline Hard $(n=27)$ & 3 & 24 & \\
\hline \multicolumn{4}{|l|}{ Laboratory findings (mean \pm SD) } \\
\hline $\mathrm{Hb}(\mathrm{g} / \mathrm{dL})$ & $12.7 \pm 1.9$ & $12.4 \pm 1.9$ & 0.58 \\
\hline 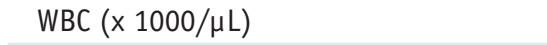 & $8.1 \pm 2.6$ & $8.2 \pm 3.1$ & 0.94 \\
\hline Platelets (x 1000/ $\mu \mathrm{L})$ & $240.4 \pm 79.0$ & $255.5 \pm 94.2$ & 0.59 \\
\hline INR & $1.0 \pm 0.1$ & $1.5 \pm 2.2$ & $<0.01$ \\
\hline $\mathrm{Na}(\mathrm{mmol} / \mathrm{L})$ & $136.2 \pm 4.1$ & $137.4 \pm 3.4$ & 0.41 \\
\hline $\mathrm{K}(\mathrm{mmol} / \mathrm{L})$ & $4.2 \pm 0.4$ & $4.2 \pm 0.6$ & 0.90 \\
\hline Urea $(\mathrm{mg} / \mathrm{dL})$ & $4.5 \pm 2.4$ & $4.8 \pm 3.7$ & 0.86 \\
\hline $\mathrm{Cr}(\mathrm{mg} / \mathrm{dL})$ & $0.8 \pm 0.4$ & $0.8 \pm 0.2$ & 0.46 \\
\hline Bilirubin (mg/dL) & $8.2 \pm 7.1$ & $4.1 \pm 5.2$ & 0.03 \\
\hline $\operatorname{ALP}(U / L)$ & $463.8 \pm 370.7$ & $222.8 \pm 198.0$ & 0.03 \\
\hline Albumin (g/dL) & $3.7 \pm 0.4$ & $3.8 \pm 0.4$ & 0.52 \\
\hline
\end{tabular}

$p<0.05$ indicates significant difference between groups. Unless otherwise stated, data are numbers of patients. ${ }^{*} 0$ thers included lymphoma, colon cancer, advanced gastric cancer, solid pseudopapillary tumor, duodenal perforation. ALP = alkaline phosphatase, CBD = common bile duct, GIST = gastrointestinal stromal tumor, INR = international normalized ratio, IPMN = intraductal papillary mucinous neoplasm, $\mathrm{PF}=$ pancreatic fistula, $\mathrm{SD}=$ standard deviation 


\section{RESULTS}

\section{Patient Demographics and CT Imaging Features}

Patient demographics and clinical laboratory results are summarized in Table 1. Twenty-nine patients had no PFs and 10 patients had PFs that were classified as grade BL. Fifteen patients $(27.8 \%)$ had clinically relevant PFs. All 15 patients with PF had grade B PFs, and no patients had grade C PFs. There were no significant differences between the PF and non-PF groups with respect to age, sex, diagnosis, or body mass index. Lower international normalized ratio $(p<0.01)$ and higher bilirubin $(p=0.03)$ and alkaline phosphatase $(p=0.03)$ levels were associated with PF occurrence. However, no additional blood test results were related to PF occurrence. The other imaging features examined were insignificant. Palpation by a surgeon showed a significant effect for predicting PF (Table 1).

\section{Reliability of HUHA and Mean CTHU Measurements \\ Bland-Altman plots of HUHA and mean CTHU} measurements are shown in Figure 3. The mean differences (95\% limits of agreement) were $0.3 \%(-10.5-11.0 \%)$ for HUHA-A (Fig. 3A), 1.6\% (-59.0-62.2\%) for HUHA-B (Fig. $3 B),-0.4 \%(-51.3-50.5 \%)$ for HUHA-C (Fig. 3C), and 1.7 HU (-18.0-21.4 HU) for mean CTHU ( $p>0.05)$ (Fig. 3D).

\section{HUHA and Mean CTHU Performance in Predicting PF Occurrence and Pancreatic Softness}

The HUHA and mean CTHU values of the PF and non-PF groups are summarized in Tables 2 and 3. PF occurrence correlation analysis showed a strong positive correlation of HUHA-A with PF ( $r=0.60 ; 95 \%$ CI, 0.47 to $0.71 ; p<$

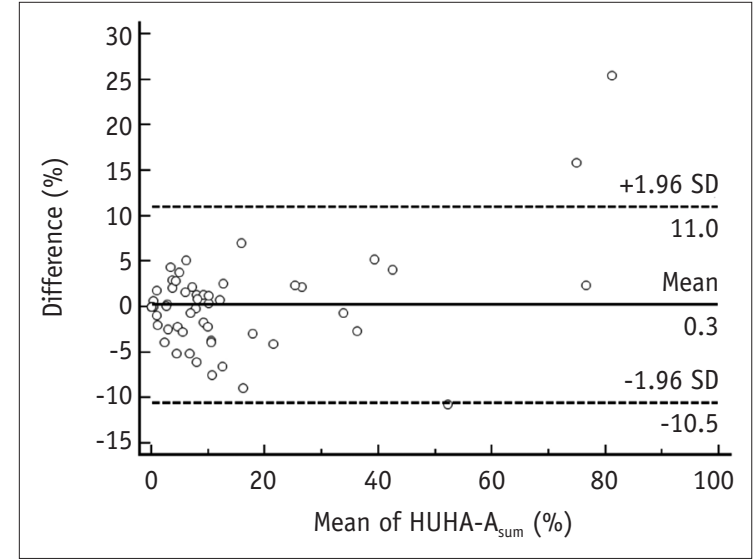

A

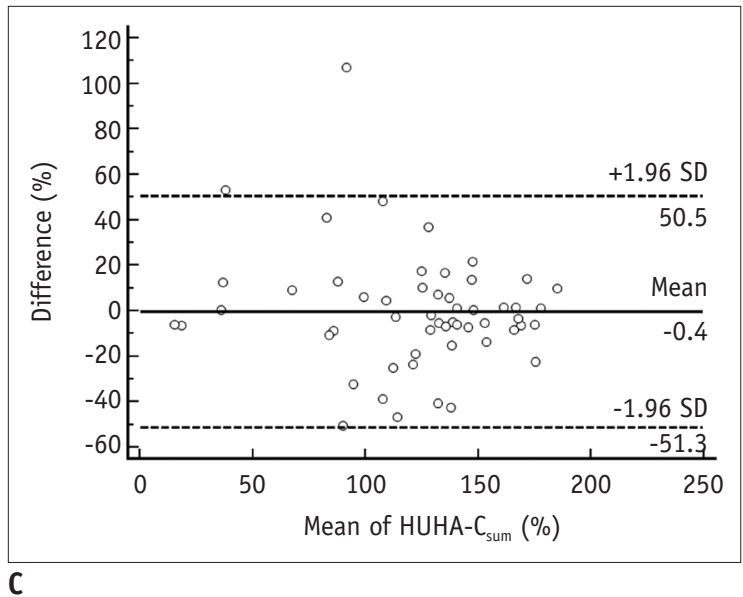

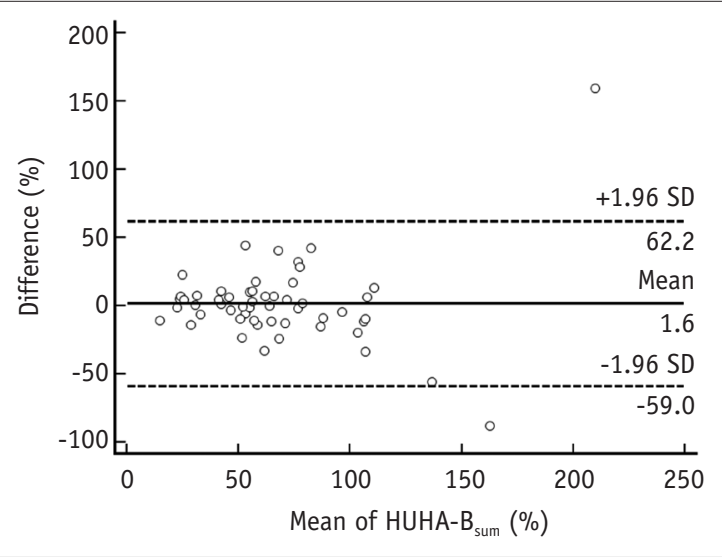

B

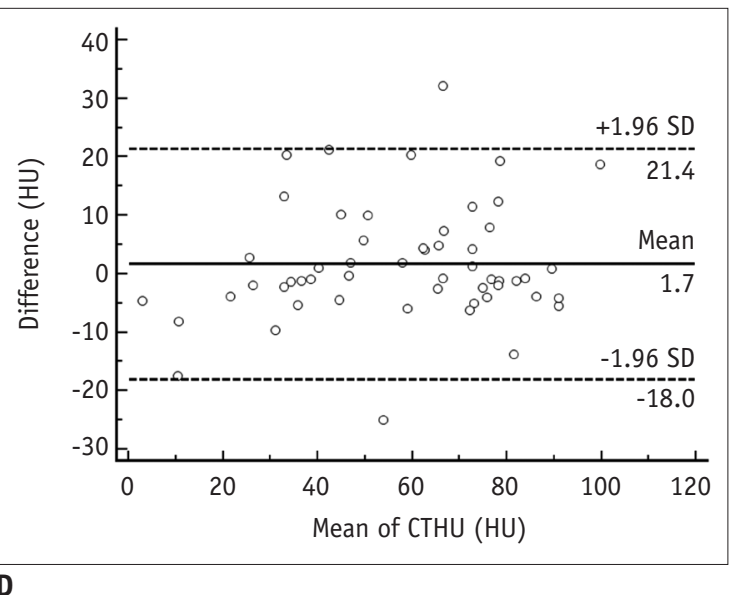

Fig. 3. Bland-Altman plots of inter-observer agreement for HUHA and mean CTHU measurements.

A-D. Differences (y axis) between two observers are plotted against mean value (x axis) of their measurements. $95 \%$ limits of agreements of HUHA-A (A), HUHA-B (B), HUHA-C (C), and mean CTHU (D) ranged from $-10.5 \%$ to $11 \%$ (mean, $0.3 \%$ ), from $-59 \%$ to $62.2 \%$ (mean, $1.6 \%$ ), from $-51.3 \%$ to $50.5 \%$ (mean $-0.4 \%$ ), and from $-18.0 \mathrm{HU}$ to $21.4 \mathrm{HU}$ (mean $1.7 \mathrm{HU}$ ), respectively. Solid line indicates mean difference. Top and bottom dashed lines correspond to upper and lower margins of $95 \%$ limits of agreement. With probability of 95\%, differences in normalized scores of future examinations will be between upper and lower limits of agreement (mean \pm variability estimate $=1.96 \times$ SD). 
Table 2. Comparison of HUHA and Mean CTHU Values between PF and Non-PF Groups for Predicting PF Occurrence

\begin{tabular}{|c|c|c|c|c|c|c|}
\hline & & iewer 1 & & & iewer 2 & \\
\hline & $P F(n=15)$ & Non-PF $(n=39)$ & $P$ & $P F(n=15)$ & Non-PF $(n=39)$ & $P$ \\
\hline HUHA-A (\%) & $16.9 \pm 14.9$ & $3.7 \pm 4.1$ & $<0.001$ & $16.3 \pm 12.0$ & $3.8 \pm 4.0$ & $<0.001$ \\
\hline HUHA-B (\%) & $42.6 \pm 31.6$ & $30.5 \pm 13.3$ & 0.14 & $43.9 \pm 22.5$ & $28.9 \pm 15.1$ & 0.03 \\
\hline HUHA-C (\%) & $50.1 \pm 26.9$ & $64.9 \pm 16.0$ & 0.04 & $44.4 \pm 25.1$ & $67.4 \pm 17.6$ & $<0.001$ \\
\hline Mean CTHU (HU) & $49.1 \pm 29.8$ & $62.0 \pm 21.5$ & 0.01 & $45.3 \pm 26.2$ & $61.1 \pm 20.3$ & $<0.001$ \\
\hline
\end{tabular}

Unless otherwise stated, data are mean \pm SD. CTHU = computed tomography Hounsfield unit, HUHA = Hounsfield unit histogram analysis

Table 3. Comparison of HUHA and Mean CTHU Values between PF and Non-PF Groups for Predicting Pancreatic Softness according to Palpation by Surgeon

\begin{tabular}{lccccccc}
\hline & \multicolumn{3}{c}{ Reviewer 1} & & \multicolumn{3}{c}{ Reviewer 2 } \\
\cline { 2 - 3 } & Hard $(\mathrm{n}=27)$ & Soft $(\mathrm{n}=27)$ & $P$ & & Hard $(\mathrm{n}=27)$ & Soft $(\mathrm{n}=27)$ & $P$ \\
\hline HUHA-A (\%) & $2.1 \pm 2.8$ & $12.7 \pm 12.5$ & $<0.001$ & & $2.7 \pm 2.3$ & $11.8 \pm 10.9$ & $<0.001$ \\
HUHA-B (\%) & $28.0 \pm 12.6$ & $39.8 \pm 25.1$ & $<0.001$ & & $25.0 \pm 10.6$ & $41.1 \pm 21.3$ & $<0.001$ \\
HUHA-C (\%) & $69.3 \pm 13.7$ & $52.4 \pm 22.8$ & $<0.001$ & & $72.3 \pm 11.7$ & $49.7 \pm 24.7$ & $<0.001$ \\
Mean CTHU (HU) & $64.2 \pm 22.5$ & $52.6 \pm 25.5$ & 0.52 & & $63.6 \pm 20.1$ & $49.9 \pm 24.0$ & 0.03 \\
\hline
\end{tabular}

Unless otherwise stated, data are mean \pm SD.

0.01). HUHA-B ( $r=0.32 ; 95 \%$ CI, 0.18 to $0.51 ; p<0.01)$, HUHA-C $(r=-0.39 ; 95 \% \mathrm{CI},-0.60$ to $-0.30 ; p<0.01)$, and mean CTHU $(r=-0.27 ; 95 \%$ CI, -0.44 to $-0.08 ; p<0.01)$ showed either weak positive or negative correlations with PF. Pancreatic softness correlation analysis showed that HUHA-A $(r=0.51 ; 95 \%$ CI, 0.36 to $0.64 ; p<0.01)$ and HUHA-C $(r=-0.47 ; 95 \%$ CI, -0.60 to $-0.30 ; p<0.01)$ had moderate correlations with pancreatic softness. HUHA-B ( $r=$ $0.36 ; 95 \%$ CI, 0.18 to $0.51 ; p<0.01)$ and mean CTHU ( $r=$ $-0.27 ; 95 \%$ CI,-0.44 to $-0.08 ; p<0.01)$ showed only weak correlations.

The ROC analysis to predict PF occurrence resulted in AUC values for HUHA-A, HUHA-B, HUHA-C, and mean CTHU of 0.86 ( $95 \%$ CI: 0.78 to 0.92 ), 0.67 (95\% CI: 0.57 to 0.76 ), 0.71 (95\% CI: 0.61 to 0.79 ), and 0.65 (95\% CI: 0.55 to $0.74)$, respectively. HUHA-A had significantly better PF predictive capabilities compared to the other values $(p<$ 0.01 ) (Fig. 4). When the $27.8 \%$ PF prevalence rate of our study population and a $>7.9 \%$ HUHA-A cutoff were applied, the sensitivity and specificity of the HUHA-A value for predicting PF were $66.7 \%$ and $92.3 \%$, respectively.

The ROC analysis to predict pancreatic softness resulted in AUC values for HUHA-A, HUHA-B, HUHA-C, and mean CTHU of 0.86 (95\% CI: 0.78 to 0.92 ), 0.71 (95\% CI: 0.61 to 0.79 ), 0.74 (95\% CI: 0.65 to 0.82 ), and 0.64 (95\% CI: 0.54 to 0.73 ), respectively (Fig. 5).

\section{DISCUSSION}

Our study demonstrated a strong positive correlation

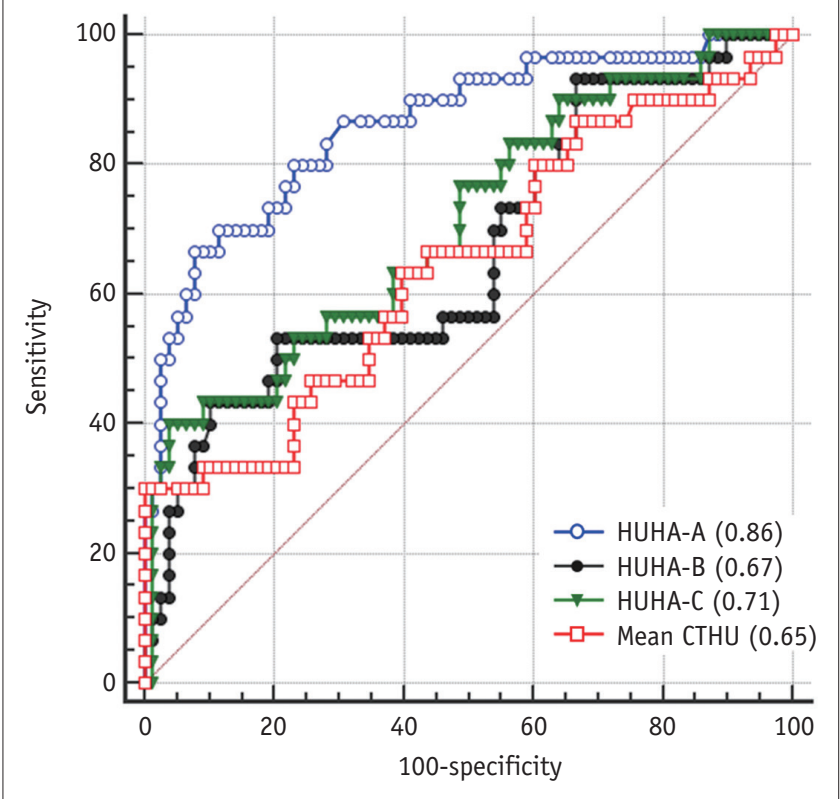

Fig. 4. ROC curve to predict PF using HUHA-A ( $A U C=0.86$ ), HUHA-B (AUC $=0.67)$, HUHA-C (AUC $=0.71)$, and mean CTHU $(A \cup C=0.65)$ values. $A U C=$ area under curve, $R O C=$ receiver operating characteristic

between the HUHA-A value and PF occurrence. Moreover, in the ROC analysis, the HUHA-A value showed good diagnostic performance in predicting PF occurrence. Since we hypothesized that the HUHA-A value represents the distribution of fat in the pancreatic parenchyma, our results suggest that the amount of fat in the pancreatic parenchyma is closely related to PF occurrence. Based on our results, the assessment of the fat content of pancreatic tissue with HUHA may be helpful in predicting 


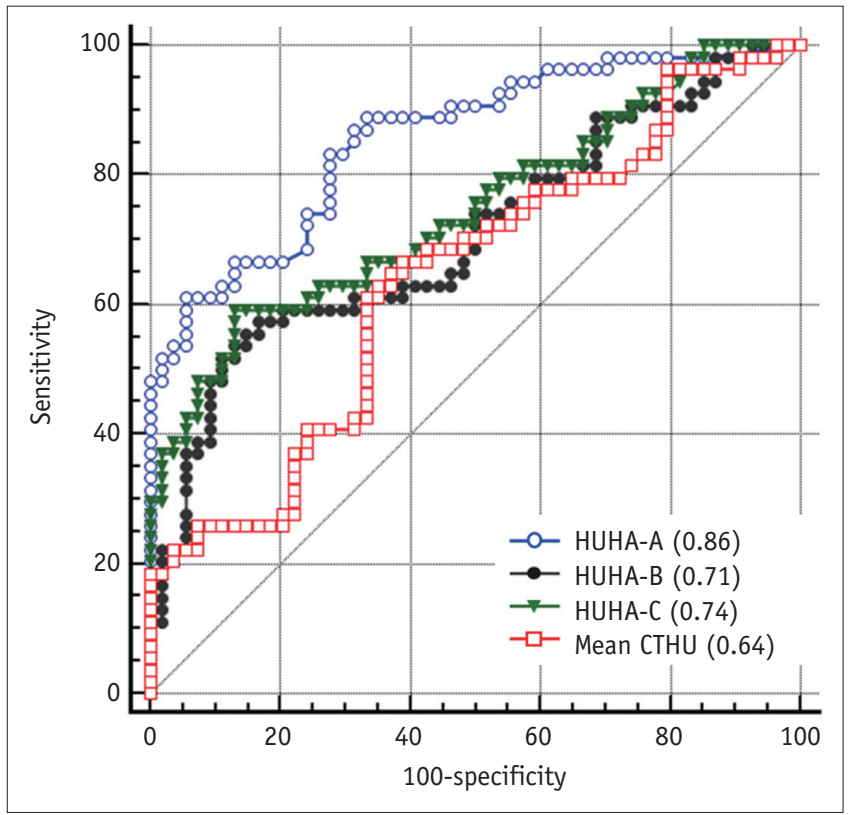

Fig. 5. ROC curve to predict pancreatic softness using HUHA-A $(A \cup C=0.86)$, HUHA-B $(A \cup C=0.71)$, HUHA-C $(A \cup C=0.74)$, and mean CTHU (AUC $=0.64)$ values.

PF occurrence and identifying patients with a high risk of developing PF. Additionally, in the ROC curve for pancreatic softness, the HUHA-A value showed a moderate correlation with pancreatic softness and had good predictive performance. As in other reports $(14,15,20)$, the mean CTHU, which is commonly used to analyze CT images, was significantly different between the PF and non-PF groups in our study. However, it showed a weak negative correlation with PF and poor diagnostic accuracy for PF prediction. Fatty changes in the pancreatic parenchyma are indicated by decreased HU values on CT. However, since the mean CTHU value is the average value of all tissues, it has limitations with respect to accurately evaluating fat. Moreover, to use the mean CTHU value to measure fat in the liver or pancreas, a calibration process using spleen or main portal vein attenuation values may be required $(15,23-$ 25). Recent advances in imaging analysis technology have made it possible to analyze the CTHU value for specific HU ranges and calculate the distribution of arbitrarily set HU ranges as a percentage. Specifically, this method can only measure the distribution of negative $\mathrm{HU}$ values representing fat content (16). In this study, we assumed that negative $\mathrm{HU}$ values represented fat tissue in the pancreas more accurately than the mean CTHU value. Our results suggest that the HUHA value represented the amount of fat within the ROI more accurately than the mean CTHU.

Fatty infiltration of the pancreas and pancreatic softness are considered important risk factors for $\operatorname{PF}(9,11,13)$. Pathologically proven fatty changes in the pancreas have been highly correlated with $\mathrm{CT}$ and magnetic resonance imaging (MRI) features. Yardimci et al. (15) calculated the pancreatic attenuation index by dividing pancreas density by spleen density. Patients with a lower pancreatic attenuation index developed PF at a significantly higher rate. Lee et al. (12) and Yoon et al. (26) demonstrated the effectiveness of using MRI to quantify pancreatic fat and reported a correlation between fatty infiltration of the pancreas and PF occurrence. In this study, we measured mean CTHU and HUHA values to determine whether they were associated with PF occurrence and found that HUHA-A was highly correlated with PF occurrence as in other studies.

Although the assessment of pancreatic softness via manual palpation by a surgeon is subjective, it has been reported to be a useful predictor of PF and, specifically, ISGPF grade C PF $(17,18,27,28)$. Similarly, our study found that PF occurred at a significantly higher rate in soft compared to hard pancreatic tissues. Furthermore, higher HUHA-A values were associated with a greater likelihood of pancreatic softness. Therefore, pancreatic softness showed a moderately positive correlation with the HUHA-A value.

Obesity, perirenal fat and pancreatic thickness, and a MPD diameter $<3 \mathrm{~mm}$ are predictive factors of PF occurrence (6, $11,13,17,18,20,27,29)$. However, in our study, none of these factors were associated with PF occurrence. Several studies have evaluated the association between a small MPD diameter and PF occurrence and had contradictory findings; however, studies enrolling more than 500 subjects found that a small MPD diameter was correlated with PF occurrence $(17,27)$. In our study, the MPD diameter was underestimated since there was no difference in the distribution of MPD diameter given the small number of patients enrolled. Pancreatic thickness is an imaging feature associated with PF occurrence. However, in previous studies, the pancreatic thickness cutoff has varied from 12 $\mathrm{mm}$ to $17.6 \mathrm{~mm}$ (29-31). Although pancreatic thickness was greater in the non-PF group in our study, there was no significant difference between the two groups. The reason for this discrepancy is unclear; however, it may have been due to our small sample size, patient body morphology, measurement methods, or observers. In particular, surgical skill or technique may have impacted PF occurrence outcomes; however, this is unlikely since a single surgeon performed all of the assessments in our study. Thus, the relationship between pancreatic thickness and PF occurrence 
requires further investigation.

HU measurement is significantly affected by intravenous contrast material (32). To avoid contrast material effects, we drew ROIs on pre-contrast CT images. Fat measurement on pre-contrast $\mathrm{CT}$ images is less affected by the $\mathrm{X}$-ray tube voltage. Bone and iodine with a high atomic number result in significant differences in mass attenuation coefficient values according to $\mathrm{X}$-ray tube voltages; however, on precontrast imaging, there is less difference in tissues, such as fat, muscle, and water. Thus, HU values measured on precontrast images may be less affected by $100-k V p$ or $120-k V p$ tube voltage (33).

Measuring HUHA values has several advantages. First, it can be measured using $\mathrm{CT}$, which is the most common and basic imaging modality used to evaluate cancer stages, operability, and comorbidities (34). Second, HUHA can be easily performed by drawing an ROI around the pancreatic parenchyma on preoperative $\mathrm{CT}$ images. During aging, the pancreas shows non-homogeneous fatty degeneration. To exclude this effect, we performed HUHA by measuring CT attenuation in the axial and corresponding sagittal planes around the pancreaticojejunostomy site with reference to postoperative images. This approach assumes that the 3D distribution of pancreatic fat along the pancreatic resection plane is better reflected by using both axial and sagittal images for measurements. The ROI location used for the HUHA was near the SMV with reference to postoperative images and generally corresponded to the pancreatic resection margin during PD. Third, by simply drawing ROIs on pre-contrast images, the HUHA provides a semiquantitative measurement of fat content as a percentage value with high inter-observer agreement. We were very careful to exclude peripancreatic retroperitoneal fat tissue from the ROIs, which can affect the results. Furthermore, for convenience, we drew circular ROIs in the pancreatic parenchyma.

Despite the interesting results, this study has certain limitations. First, given the retrospective nature of this study, potential selection bias was inevitable. Second, the degree of fatty infiltration and pancreatic fibrosis was not evaluated histologically. However, several CT studies have demonstrated the effectiveness of fat quantification for determining pathological predictors of PF occurrence. Third, our study population was relatively small and patients had various pathological diagnoses, which could have been a confounding factor. Moreover, the study did not have any cases of ISGPF grade C. Fourth, HUHA values were categorized into 3 groups arbitrarily. Thus, a larger cohort study and external validation are required. Lastly, although CT scanners are regularly calibrated according to the manufacturer's guidelines, attenuation values may differ based on X-ray beam properties. Therefore, the HU value will differ depending on the CT scanners and protocols used.

In conclusion, the HUHA-A values on preoperative precontrast $\mathrm{CT}$ images demonstrate a strong correlation with PF occurrence and is effective in predicting PF occurrence.

\section{Conflicts of Interest}

The authors have no potential conflicts of interest to disclose.

\section{Acknowledgments}

The English in this document has been checked by at least two professional editors, both native speakers of English. For a certificate, please see: http://www.textcheck.com/certificate/AS8zDf

\section{ORCID iDs}

Hong Il $\mathrm{Ha}$

https://orcid.org/0000-0001-9050-7486

Wonju Hong

https://orcid.org/0000-0001-6891-5483

Jung Woo Lee

https://orcid.org/0000-0001-6942-9655

Sang Min Lee

https://orcid.org/0000-0001-7719-3849

Min-Jeong Kim

https://orcid.org/0000-0002-7484-5896

\section{REFERENCES}

1. Lerut JP, Gianello PR, Otte JB, Kestens PJ. Pancreaticoduodenal resection. Surgical experience and evaluation of risk factors in 103 patients. Ann Surg 1984;199:432-437

2. Yeo CJ, Cameron JL, Sohn TA, Lillemoe KD, Pitt HA, Talamini MA, et al. Six hundred fifty consecutive pancreaticoduodenectomies in the 1990s: pathology, complications, and outcomes. Ann Surg 1997;226:248-260; discussion 257-260

3. Seo JW, Hwang HK, Lee M, Kim KW, Kang CM, Kim MJ, et al. Normal postoperative computed tomography findings after a variety of pancreatic surgeries. Korean J Radiol 2017;18:299308

4. Miedema BW, Sarr MG, van Heerden JA, Nagorney DM, McIlrath DC, Ilstrup D. Complications following pancreaticoduodenectomy. Current management. Arch Surg 
1992;127:945-950; discussion 949-950

5. Vanounou T, Pratt WB, Callery MP, Vollmer CM Jr. Selective administration of prophylactic octreotide during pancreaticoduodenectomy: a clinical and cost-benefit analysis in low- and high-risk glands. J Am Coll Surg 2007;205:546557

6. Roberts KJ, Storey R, Hodson J, Smith AM, Morris-Stiff G. Pre-operative prediction of pancreatic fistula: is it possible? Pancreatology 2013;13:423-428

7. van Berge Henegouwen MI, De Wit LT, Van Gulik TM, Obertop $H$, Gouma DJ. Incidence, risk factors, and treatment of pancreatic leakage after pancreaticoduodenectomy: drainage versus resection of the pancreatic remnant. J Am Coll Surg 1997;185:18-24

8. Bassi C, Dervenis C, Butturini G, Fingerhut A, Yeo C, Izbicki J, et al. Postoperative pancreatic fistula: an international study group (ISGPF) definition. Surgery 2005;138:8-13

9. Mathur A, Pitt HA, Marine M, Saxena R, Schmidt CM, Howard $\mathrm{TJ}$, et al. Fatty pancreas: a factor in postoperative pancreatic fistula. Ann Surg 2007;246:1058-1064

10. Bassi C, Marchegiani G, Dervenis C, Sarr M, Abu Hilal M, Adham M, et al. The 2016 update of the international study group (ISGPS) definition and grading of postoperative pancreatic fistula: 11 years after. Surgery 2017;161:584-591

11. Rosso E, Casnedi S, Pessaux P, Oussoultzoglou E, Panaro $F$, Mahfud M, et al. The role of "fatty pancreas" and of BMI in the occurrence of pancreatic fistula after pancreaticoduodenectomy. J Gastrointest Surg 2009;13:18451851

12. Lee SE, Jang JY, Lim CS, Kang MJ, Kim SH, Kim MA, et al. Measurement of pancreatic fat by magnetic resonance imaging: predicting the occurrence of pancreatic fistula after pancreatoduodenectomy. Ann Surg 2010;251:932-936

13. Sugimoto M, Takahashi S, Kojima M, Kobayashi T, Gotohda $\mathrm{N}$, Konishi M. In patients with a soft pancreas, a thick parenchyma, a small duct, and fatty infiltration are significant risks for pancreatic fistula after pancreaticoduodenectomy. $J$ Gastrointest Surg 2017;21:846-854

14. Machado NO. Pancreatic fistula after pancreatectomy: definitions, risk factors, preventive measures, and management-review. Int J Surg Oncol 2012;2012:602478

15. Yardimci S, Kara YB, Tuney D, Attaallah W, Ugurlu MU, Dulundu $E$, et al. A simple method to evaluate whether pancreas texture can be used to predict pancreatic fistula risk after pancreatoduodenectomy. J Gastrointest Surg 2015;19:1625-1631

16. Lim HK, Ha HI, Park SY, Lee K. Comparison of the diagnostic performance of CT Hounsfield unit histogram analysis and dual-energy $\mathrm{X}$-ray absorptiometry in predicting osteoporosis of the femur. Eur Radiol 2018 Sep 25 [Epub ahead of print]. https://doi.org/10.1007/s00330-018-5728-0

17. Hu BY, Wan T, Zhang WZ, Dong JH. Risk factors for postoperative pancreatic fistula: analysis of 539 successive cases of pancreaticoduodenectomy. World J Gastroenterol
2016;22:7797-7805

18. McMillan MT, Vollmer CM Jr, Asbun HJ, Ball CG, Bassi C, Beane JD, et al. The characterization and prediction of ISGPF grade C fistulas following pancreatoduodenectomy. J Gastrointest Surg 2016;20:262-276

19. Hashimoto Y, Sclabas GM, Takahashi N, Kirihara Y, Smyrk TC, Huebner M, et al. Dual-phase computed tomography for assessment of pancreatic fibrosis and anastomotic failure risk following pancreatoduodenectomy. J Gastrointest Surg 2011;15:2193-2204

20. Frozanpor F, Loizou L, Ansorge C, Lundell L, Albiin $N$, Segersvärd R. Correlation between preoperative imaging and intraoperative risk assessment in the prediction of postoperative pancreatic fistula following pancreatoduodenectomy. World J Surg 2014;38:2422-2429

21. DeLong ER, DeLong DM, Clarke-Pearson DL. Comparing the areas under two or more correlated receiver operating characteristic curves: a nonparametric approach. Biometrics 1988;44:837-845

22. Park SH, Goo JM, Jo CH. Receiver operating characteristic (ROC) curve: practical review for radiologists. Korean J Radiol 2004;5:11-18

23. Kim SY, Kim H, Cho JY, Lim S, Cha K, Lee KH, et al. Quantitative assessment of pancreatic fat by using unenhanced CT: pathologic correlation and clinical implications. Radiology 2014;271:104-112

24. Pickhardt PJ, Graffy PM, Reeder SB, Hernando D, Li K. Quantification of liver fat content with unenhanced MDCT: phantom and clinical correlation with MRI proton density fat fraction. AJR Am J Roentgenol 2018;211:W151-W157

25. Kramer $\mathrm{H}$, Pickhardt PJ, Kliewer MA, Hernando D, Chen GH, Zagzebski JA, et al. Accuracy of liver fat quantification with advanced CT, MRI, and ultrasound techniques: prospective comparison with MR spectroscopy. AJR Am J Roentgenol 2017;208:92-100

26. Yoon JH, Lee JM, Lee KB, Kim SW, Kang MJ, Jang JY, et al. Pancreatic steatosis and fibrosis: quantitative assessment with preoperative multiparametric MR imaging. Radiology 2016;279:140-150

27. Kawai M, Kondo S, Yamaue H, Wada K, Sano K, Motoi F, et al. Predictive risk factors for clinically relevant pancreatic fistula analyzed in 1,239 patients with pancreaticoduodenectomy: multicenter data collection as a project study of pancreatic surgery by the Japanese Society of Hepato-Biliary-Pancreatic Surgery. J Hepatobiliary Pancreat Sci 2011;18:601-608

28. Sugimoto M, Takahashi S, Kojima M, Gotohda N, Kato $Y$, Kawano $S$, et al. What is the nature of pancreatic consistency? Assessment of the elastic modulus of the pancreas and comparison with tactile sensation, histology, and occurrence of postoperative pancreatic fistula after pancreaticoduodenectomy. Surgery 2014;156:1204-1211

29. Chang YR, Kang JS, Jang JY, Jung WH, Kang MJ, Lee KB, et al. Prediction of pancreatic fistula after distal pancreatectomy based on cross-sectional images. World J Surg 2017;41:1610- 
1617

30. Sugimoto M, Takahashi S, Gotohda N, Kato Y, Kinoshita T, Shibasaki $\mathrm{H}$, et al. Schematic pancreatic configuration: a risk assessment for postoperative pancreatic fistula after pancreaticoduodenectomy. J Gastrointest Surg 2013;17:17441751

31. Okano K, Oshima M, Kakinoki K, Yamamoto N, Akamoto S, Yachida S, et al. Pancreatic thickness as a predictive factor for postoperative pancreatic fistula after distal pancreatectomy using an endopath stapler. Surg Today 2013;43:141-147

32. Pompe E, Willemink MJ, Dijkhuis GR, Verhaar HJ, Mohamed
Hoesein FA, de Jong PA. Intravenous contrast injection significantly affects bone mineral density measured on CT. Eur Radiol 2015;25:283-289

33. Bushberg JT, Seibert JA, Leidholdt Jr. EM, Boone JM. The essential physics of medical imaging, 3rd ed. Philadelphia, PA: Wolters Kluwer Health/Lippincott Williams and Wilkins, 2012:324

34. Tempero MA, Arnoletti JP, Behrman SW, Ben-Josef E, Benson $A B$ 3rd, Casper ES, et al. Pancreatic Adenocarcinoma, version 2.2012: featured updates to the NCCN Guidelines. J Natl Compr Canc Netw 2012;10:703-713 